\title{
MYKHAILO PAVLOVYCH LISITSA (to the 100th anniversary of his birth)
}

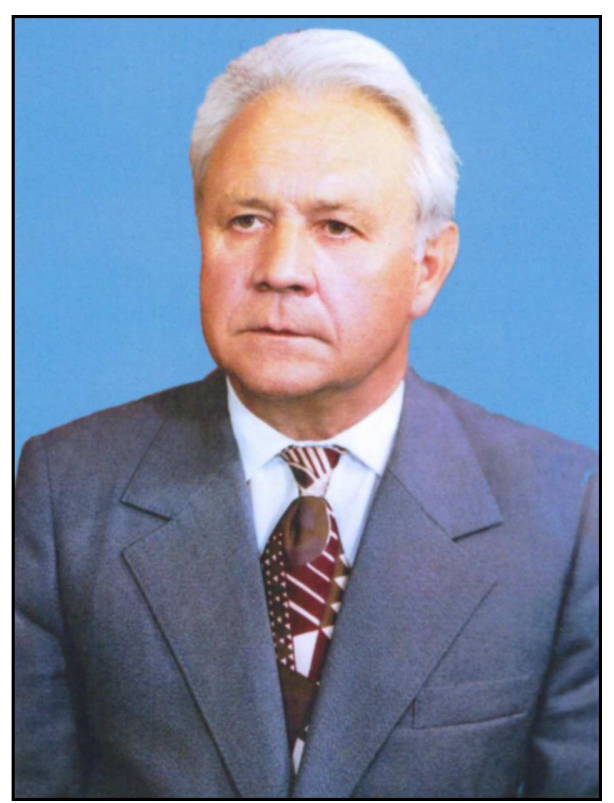

January 15, 2021, marks the 100th anniversary of the birthday of Academician of the National Academy of Sciences of Ukraine (NASU) Mykhailo Pavlovych Lisitsa, the prominent Ukrainian scientist in the fields of optics and solid state physics, a talented teacher who created a powerful scientific school of solid state spectroscopy. The results of M.P. Lisitsa made a significant contribution to spectroscopy, nonlinear optics, quantum electronics, solid state physics, and, in particular, semiconductor physics.

M.P. Lisitsa was born in the village of Vysoke (the Zhytomyr region) in a peasant family. In 1938, he graduated from the Kyiv pedagogical technical school and continued his studies by entering the Faculty of Physics and Mathematics at T.G. Shevchenko Kyiv State University (KSU). However, two months later, he was drafted into the army. M.P. Lisitsa was in the army till October 1945. During World War II, he was a regiment scout and many times went behind the front line to capture a prisoner for interrogation. For his courage and bravery, he was awarded three orders and many medals including a medal "For Courage".
After the war, M.P. Lisitsa continued his studies at the Faculty of Physics of the KSU. After graduation, he became a graduate student at the Department of Optics. The Ph.D. thesis of Mykhailo Pavlovych (1954) was the first work in the world devoted to the study of vibrational spectra of molecular microcrystals in polarized light. To perform his research, the graduate student Lisitsa had had to create the unique, at that time, microlens with mirror optics, as well as multilayer polarizers transparent to infrared radiation. In addition, he developed a method for calculating the parameters of layered systems on the basis of obtained experimental results. Based on his research works, M.P. Lisitsa experimentally proved the applicability of the theory of molecular excitons to the vibrational spectra of molecules.

The talented young researcher was committed to lecturing the basic general course of physics at the faculties of physics and chemistry (all 6 semesters), as well as several special courses in spectroscopy, group theory, quantum mechanics, and nonlinear optics at the faculties of physics and radiophysics. The extraordinary talent of Mykhailo Pavlovych as a lecturer manifested itself when he elaborated the structure of the courses and when he brightly and skilfully presented the lecture material. His lectures invoked interest in science in his students and encouraged them to do research under his guidance. The foundations of M.P. Lisitsa's scientific school were laid in the following years.

In 1955-1961, Mykhailo Pavlovych together with his graduate students were solving the problem of determining the temperature dependences of the spectra of normal vibrations for molecular compounds in the gaseous, liquid, and solid states. The corresponding cycle of scientific publications formed a basis of the doctoral dissertation of M.P. Lisitsa (1961). Afterwards, those studies were continued, and they led to the discovery and substantiation of a new phenomenon, the combined Fermi-Davydov resonance.

In 1961, the scientist transferred to the Institute of Semiconductor Physics of the Academy of Sciences 
of the UkrSSR, took the position of the head of the Department of optics, and continued to work parttime as a professor at the Department of Physics of the KSU. It was the management of the department where the organizational talent of Mykhailo Pavlovych revealed itself to the full extent. It concerned the formation of a friendly and powerful scientific team composed of like-minded people, as well as the ability of Mykhailo Pavlovych to anticipate new directions in the development of physics and to choose the most promising of them. One of such directions was connected with the development of new methods aimed at studying the properties of substances with the help of lasers, which were, at that time, just invented exotic sources for the optical excitation. The department headed by M.P. Lisitsa immediately took the leading position in the study of nonlinear properties of crystals. Several important results were obtained in this sphere under the supervision of Mykhailo Pavlovych. Among them, e.g., the effect of exciton disappearance at high concentrations of photogenerated charge carriers and the emergence of an electron-hole plasma, as well as the effect of interband absorption saturation and abrupt switching into the induced transparency mode in colored glass filters under the action of intense laser light (as well as the method of passive Q-switching that was proposed on its basis), should be mentioned. Besides, the nature of dominant mechanisms giving rise to the optical destruction of the surface and bulk of transparent insulators and semiconductors was studied, and the destruction thresholds for alkali-halide crystals and semiconductors of the $\mathrm{A}^{2} \mathrm{~B}^{6}, \mathrm{~A}^{2} \mathrm{~B}^{5}$, and $\mathrm{A}^{5} \mathrm{~B}^{6}$ types were determined. On the basis of the results obtained, while studying the two-photon absorption and polarization effects in various crystals, the application of nonlinearly absorbing and optically active semiconductor wafers was proposed to restrict the power of laser beams, as well as to stabilize and correct their space-time intensity distribution. Finally, two new nonlinear optical polarization phenomena were experimentally discovered: an additional nonlinear optical activity in gyrotropic crystals and a principally new giant optical activity in nonhyrotropic cubic crystals with impurity tunnel centers.

Another example of M.P. Lisitsa's ability to "look beyond the scientific horizons" is a scientific direction initiated by him to study quasi-zero-dimensional crys- tals, which had been actively developed for almost 20 years before the appearance of nanophysics, one of the most popular directions in modern physics. Such examples also include Mykhailo Pavlovych's passion for the physics of living organisms. He analyzed the resonant character of the interaction of millimeterwavelength electromagnetic waves with living organisms, in particular, with the human body. As a result, he identified those quantum transitions of the vibrational, rotational, inversion, and spin nature that possess therapeutic effects, if those waves are applied to irradiate acupuncture points along the meridian associated with a sick organ.

Other classical directions of research were also successfully developed at the department of semiconductor optics. In particular, phonon excitations in semiconductor crystals were actively studied. The essential role of various mechanisms of a resonant interaction with the participation of phonon excitations giving rise to the appearance of peculiarities in the spectra of pure, doped, and mixed semiconductors was established. A new approach was proposed to the interpretation of phonon spectra of crystals with a layered structure. Methods for a substantial enhancement of the sensitivity of the optical diagnostics of semiconductor parameters with the help of resonance Raman scattering were elaborated.

Under the influence of M.P. Lisitsa, the application of optical methods in the new area of semiconductor research reached a new level not only at the Institute of Semiconductor Physics but also in other scientific and educational institutions of Ukraine. In particular, this occurred because Mykhailo Pavlovych's disciples had headed research departments at various institutes and universities in Ukraine. In total, M.P. Lisitsa's school includes two Corresponding Members of the NASU, 24 Dr.Sci.'s, and 50 Ph.D.'s, who continue the research in optics and spectroscopy initiated by him, including the absorption optics of the elementary and collective excitations of various types in semiconductors, luminescence studies, Raman scattering in solids, and new polarization phenomena.

The scientific heritage of Academician M.P. Lisitsa includes more than 500 scientific papers and about 40 inventor's certificates. In co-authorship with his disciples, he published 6 monographs and the 5volume edition of "Entertaining Optics".

The results of the many-year fruitful work of M.P. Lisitsa have gained deserved recognition. This 
is evidenced by awarding him the Johannes Marcus Marci Medal by the Academy of Sciences of Czechoslovakia as an outstanding spectroscopist, two State Prizes of Ukraine in science and engineering, the highest award of the NASU - the V.I. Vernadskyi Gold Medal, and the title of "Honored Worker of Science and Engineering of Ukraine". The asteroid No. 8064 Lisitsa was named after him.
The memory of the outstanding scientist and teacher is kept by his disciples, who not only continue to research in the areas indicated by him, but also start new, original directions.

O.YE. BELYAEV, I.V. BLONS'KYI, M.S. BRODYN, O.V. VAKULENKO, M.YA. VALAKH, V.O. KOCHELAP, L.V. POPERENKO, G.YU. RUDKO, F.F. SYZOV, G.G. TARASOV, V.O. YUKHYMCHUK 